\title{
Resposta de cultivares de trigo (Triticum aestivum L.) ao controle químico das principais doenças fúngicas da cultura
}

\author{
Benedito de Camargo Barros ${ }^{1}$, Jairo Lopes de Castro $^{2}$, Flávia Rodrigues Alves Patrício ${ }^{1}$
}

\author{
IInstituto Biológico, C. Postal 70, 13001-970 Campinas, SP. e-mail: bcbarros@ biologico.sp.gov.br \\ ${ }^{2}$ Pólo Regional de Desenvolvimento Tecnológico dos Agronegócios do Sudoeste Paulista \\ C. Postal 62, 18300-000, Capão Bonito, SP \\ Data de chegada: 10/12/2004. Aceito para publicação: 01/12/2005.
}

\begin{abstract}
Barros, B.C.; Castro, J.L.; Patrício, F.R.A. Response of wheat cultivars (Triticum aestivum L.) to the chemical control of fungal diseases. Summa Phytopathologica, v.32, n.3, p.239-246, 2006.

Leaf rust, leaf spot or brown spot and powdery mildew, caused by Puccinia recondita tritici, Bipolaris sorokiniana and Blumeria graminis tritici, respectively, are wheat diseases that may severely decrease crop yield. Several practices are recommended for the control of these diseases such as cultivar resistance, planting at a suitable time, and chemical control, without which the crop frequently becomes uneconomical. The objective of the present work was to evaluate, under field conditions, the response of six wheat cultivars, IAC 24, IAC 289, IAC 350, IAC 362, IAC 364 and IAC 370, in four experiments, carried out from 2000 to 2003 at Capão Bonito, State of São Paulo, Brazil. The experiments were set out in a randomized block design with four replications, and analyzed as a $2 \times 6$ factorial, being fungicide treatment (with and without) the factor 1 and cultivar the factor 2. Severity of the diseases was graded in a scale that ranged from 0 to $9,(0=$ without symptoms and $9>60 \%$ of the foliar area

affected by disease). Grain yield and the thousand kernel weight (TKW) were also assessed in each experiment. Powdery mildew was only observed in 2000, but leaf rust was detected in all years in high severity indexes (30 to $60 \%$ of leaf area affected by the disease). Leaf spot, detected in the last three years, from 2001 to 2003, showed the highest severity indexes in the rainiest year, 2001 (up to $60 \%$ of the leaf affected by the disease). In all years the fungicides were efficient to control the diseases in all experiments and resulted in higher TKW and in grain yield increases. The cultivars with the longest crop cycles, IAC 370, IAC 289 and IAC 350, had the highest responses in yield to the chemical control, despite the fact that the last two cultivars were less affected by the diseases in the control plots, showing that not only cultivar resistance, but the crop cycle and the yield potential, characteristic of each cultivar, were important in the response to the chemical control.
\end{abstract}

Additional keywords: Bipolaris sorokiniana, Puccinia recondita tritici, Blumeria graminis tritici, a thousand seed weight, yield.

\section{RESUMO}

Barros, B.C.; Castro, J.L.; Patrício, F.R.A. Resposta de cultivares de trigo (Triticum aestivum L.) ao controle químico das principais doenças fúngicas da cultura. Summa Phytopathologica, v.32, n.3, p.239-246, 2006.

Doenças do trigo, como a ferrugem-da-folha, a helmintosporiose e o oídio, causadas por Puccinia recondita, Bipolaris sorokiniana e Blumeria graminis tritici, respectivamente, podem reduzir severamente o rendimento da cultura. Diversas medidas são preconizadas para o controle das mesmas, entre as quais o emprego de cultivares resistentes, na época adequada, e o controle químico, sem o qual, freqüentemente, a cultura torna-se inviável economicamente. No presente trabalho avaliou-se, em condições de campo, a resposta a aplicações de fungicidas de seis cultivares de trigo, IAC 24, IAC 289, IAC 350, IAC 362, IAC 364 e IAC 370, em quatro experimentos instalados nos anos de 2000 a 2003, em Capão Bonito, Estado de São Paulo. Os ensaios foram realizados em delineamento em blocos ao acaso, com 4 repetições, e analisados, a cada ano, em esquema fatorial, 2 × 6 , sendo fungicida o fator 1 (com e sem) e cultivar o fator 2. As pulverizações, duas ou três, foram iniciadas quando a ferrugem-da-folha atingiu entre 10 e $15 \%$ de incidência, e repetidas após aproximadamente 15 dias. Avaliou-se a severidade das doenças por meio de uma escala de notas, que variou de 0 a 9 sendo 0 - ausência de sintomas e 9 maior que $60 \%$ de área de tecido foliar afetado pelas doenças, além do peso de mil sementes (PMS) e do rendimento. O oídio foi encontrado apenas em 2000, a ferrugem-da-folha em todos os anos em elevados índices de severidade (30 a 60\% de área foliar afetada) e a helmintosporiose 
ocorreu nos três últimos anos, 2001 a 2003, apresentando os maiores índices de severidade em 2001 (até $60 \%$ de área foliar afetada), o ano mais chuvoso. Em todos os anos os tratamentos químicos proporcionaram controle das doenças, aumentos no PMS e no rendimento. Nos anos mais favoráveis para a cultura, 2002 e 2003, o retorno em rendimento foi maior. As cultivares de ciclo mais longo,
IAC 370, IAC 289 e IAC 350, foram as que mais responderam à aplicação do fungicida, embora as duas últimas tenham apresentado os menores índices de severidade das doenças, indicando que não só a resistência, mas fatores como o ciclo e características de produtividade intrínsecas a cada cultivar podem ser importantes na resposta ao controle químico.

Palavras-chave adicionais: Bipolaris sorokiniana, Puccinia recondita tritici, Blumeria graminis tritici, peso de mil sementes, rendimento.

O trigo é uma cultura de grande importância para o Brasil, havendo uma demanda crescente da população brasileira por derivados desse cereal, estimada em 11,2 milhões de toneladas para 2005. Considerando que a produção do país, 4,5 milhões de toneladas em 2003, atende apenas parte dessa demanda, observa-se que o governo brasileiro deverá despender consideráveis esforços para suprir o mercado interno nos próximos anos (28). O país tem condições de clima, solo, material genético e tecnologia disponível para cultivar mais de 10 milhões de hectares do cereal (28).

A cultura do trigo no Brasil, assim como em outros países, pode ser afetada por várias doenças, que estão entre os principais fatores que limitam ou comprometem a sua produção. As doenças que incidem na parte aérea, como as ferrugens, o oídio, as manchas foliares e as de espiga podem causar prejuízos consideráveis, especialmente sob condições climáticas favoráveis ao desenvolvimento de epidemias. Os prejuízos são agravados quando várias doenças incidem simultaneamente na lavoura. Dados sobre danos causados por doenças na cultura do trigo são bastante variáveis, especialmente porque diversos fatores interferem no estabelecimento e desenvolvimento das epidemias, tais como as condições ambientais, a maior ou menor suscetibilidade das cultivares, a agressividade dos patógenos, a época do início da infecção, entre outros $(5,18,21)$.

A ferrugem-da-folha ou ferrugem-alaranjada, causada por Puccinia recondita Rob. ex Desm. f.sp. tritici Eriks. \& Henn., pode ser encontrada nas diferentes regiões que cultivam o trigo no Brasil, acarretando, ocasionalmente, prejuízos severos (27). Barcellos \& Ignaczak (2) relataram perdas de $50 \%$ no rendimento de grãos devido a essa doença no Rio Grande do Sul, e severas perdas no Paraná também foram mencionadas por Mehta et al. (20). Não são raras as vezes em que a extrema suscetibilidade da cultivar inviabiliza seu cultivo (3).

A mancha foliar ou helmintosporiose ou ainda a mancha marrom, cujo agente causal é Cochliobolus sativus (Ito \& Kurib.), Drechs. ex Dastur. (anamorfo: Bipolaris sorokiniana Sacc. In Sorok.) é considerada uma das doenças mais danosas para a cultura do trigo (20), chegando a acarretar perdas de 20 a $80 \%$ no rendimento desse cereal $(5,18,21)$. Sob condições muito favoráveis, a doença ocorre durante todo o ciclo da cultura (18). O agente causal ataca todas as partes da planta, do colo às espigas, sendo disseminado por meio de restos de cultura (26), plantas hospedeiras e sementes $(16,25)$. A planta torna-se mais vulnerável à infecção pelo patógeno a partir do estádio de inflorescência, em função da maior translocação de nutrientes para o desenvolvimento da panícula e o enchimento dos grãos (7). Quanto mais avançada a idade da folha bandeira maior a suscetibilidade da cultura a esse patógeno (29).

O oídio, causado pelo fungo Blumeria graminis f.sp. tritici,
(DC) E.O. Speer = Erysiphe graminis D.C. f.sp. tritici E. Marshall; (anamorfo: Oidium monilioides (Nees) Link), provoca danos significativos caso esteja presente desde os estádios iniciais da cultura do trigo, que ocorre quando prevalecem temperaturas amenas no começo do ciclo da cultura. A importância dessa doença está mais relacionada à intensidade do que à frequiência do ataque. Linhares (19) relatou perdas de 20 a 55\% no rendimento, em função da época do início do controle químico dessa doença, sendo essas perdas variáveis de acordo as condições climáticas e a cultivar plantada. Reduções de rendimento de até $64 \%$, foram quantificadas entre linhas quase isogênicas resistentes e suscetíveis de trigo (12).

O manejo das doenças do trigo preconiza a adoção simultânea de várias práticas como a utilização de cultivares resistentes e/ou precoces, o plantio em época adequada, especialmente para evitar danos causados pelas ferrugens e oídio, práticas culturais e a aplicação de fungicidas $(20,23)$. O controle químico é recomendável para culturas bem conduzidas e com alto potencial produtivo $(17,22)$, mas pode ter um importante papel na garantia da produtividade da cultura, desde que empregado com critério, dentro de um contexto que considera o ciclo biológico do patógeno, o comportamento das cultivares e as condições ambientais $(13,23)$. As epidemias de doenças foliares iniciadas antes da emergência da folha bandeira geralmente têm maior impacto sobre a produção e as recomendações de controle, visando minimizar as perdas, sugerem que sejam realizadas aplicações logo após o aparecimento dos primeiros sintomas $(4,9,20,23)$.

Alguns trabalhos mostraram diferentes respostas de cultivares de trigo ao tratamento químico $(6,11,14)$, sendo as mais suscetíveis, especialmente à ferrugem-da-folha, as que freqüentemente apresentam os maiores ganhos em produtividade após a aplicação de fungicidas $(2,6,11,14)$. Outras características inerentes às mesmas, como o ciclo, também podem ser importantes na resposta ao controle químico $(6,10)$.

Considerando que o controle químico é parte dos componentes que garantem o rendimento da cultura do trigo, especialmente em anos favoráveis a epidemias de doenças foliares, e que cultivares de trigo podem responder de maneira diversa a tratamentos com fungicidas, efetuaram-se estudos visando avaliar a resposta de seis cultivares de trigo recomendadas para o Estado de São Paulo ao tratamento com fungicidas convencionalmente empregados na cultura.

\section{MATERIALEMÉTODOS}

Quatro experimentos foram conduzidos no período de 2000 a 2003, no Pólo Regional de Desenvolvimento Tecnológico dos Agronegócios do Sudoeste Paulista (PRDTASP/APTA), em 
Capão Bonito-SP. Essa região se caracteriza por ter inverno úmido, propício para a cultura do trigo, que também pode ser favorável ao ataque de doenças foliares.

Os experimentos foram instalados em delineamento em blocos ao acaso, com quatro repetições, sendo cada parcela composta por dez linhas com $5 \mathrm{~m}$ de comprimento, com espaçamento de $0,20 \mathrm{~m}$ entre as mesmas $\left(10 \mathrm{~m}^{2}\right.$ de área útil $)$. Os experimentos foram analisados em esquema fatorial 2 × 6 , sendo fungicida o fator 1 (com e sem fungicida) e cultivar o fator 2 (IAC 24, IAC 289, IAC 350, IAC 362, IAC 364 e IAC 370). As cultivares testadas são recomendadas para o Estado de São Paulo, e apresentam características de resistência às doenças, ciclo e produtividade diferentes (22).

As semeaduras dos experimentos ocorreram entre abril e maio, época recomendada para a região Sul do Estado, excetuando-se o ano de 2002, retardada para junho, por falta de umidade no solo. A densidade de semeadura, a adubação de base e a cobertura foram realizados de acordo com as recomendações para a cultura (22).

Os seguintes fungicidas, escolhidos entre os recomendados para a cultura, com os respectivos ingredientes ativos (i. a.), concentrações (g/L), formulações e doses (g i. a./ha) foram aplicados: propiconazole, 250, concentrado emulsionável, (CE), 125, no ano de 2000, tebuconazole, 200, (CE), 150, em 2001 e 2002 e trifloxystrobin + propiconazole, 250, (CE) 150, em 2003. As aplicações foram efetuadas de acordo com as recomendações oficiais observando-se, basicamente, a ocorrência de ferrugem da folha (22). Índices de incidência da doença de 10 a $15 \%$ foram atingidos quase simultaneamente nas diferentes cultivares, sendo por esta razão as aplicações realizadas nas mesmas datas em todas as cultivares. Foram realizadas duas ou três pulverizações dependendo das condições de cada ano, sendo as primeiras efetuadas entre os estádios Z-52 e 58 (início e emergência completa da inflorescência) e as últimas entre Z-71 a Z-78 (final do florescimento a grão leitoso) da escala proposta por Zadoks et al. (30). Os fungicidas foram aplicados com pulverizador costal de pressão constante de $\mathrm{CO}_{2}$, equipado com bicos tipo D2 ${ }_{13}$, espaçados de $0,20 \mathrm{~m}$. A vazão utilizada foi de $240 \mathrm{~L} / \mathrm{ha}$, aplicada sob pressão de $60 \mathrm{lb} / \mathrm{pol}^{2}$.

As doenças ocorreram naturalmente nos experimentos e as suas severidades foram avaliadas em folhas individuais expandidas de vinte plantas por parcela, sendo baseadas na porcentagem relativa da área foliar lesionada, visualmente observada, na fase de início de maturação dos grãos, estádio de cera mole (30), com uma escala de 0 a 9 , na qual $0=$ sem sintomas; $1=1 \% ; 2=5 \% ; 3=10 \% ; 4=20 \% ; 5=30 \% ; 5=40 \% ; 7=50 \%$; $8=60 \%$ e $9=>60 \%$ de área foliar afetada, atribuindo-se finalmente uma nota média para cada parcela. Foram também determinados o rendimento ( $\mathrm{kg} / \mathrm{ha}$ ) e o peso de mil sementes (PMS).

As médias foram comparadas pelo teste de Tukey a 5\% de probabilidade, sendo também realizada a análise de correlação entre as variáveis rendimento e o PMS e as variáveis severidade da ferrugem-da-folha e da helmintosporiose.

\section{RESULTADOS E DISCUSSÃO}

A ferrugem-da-folha foi detectada em todos os anos, a helmintosporiose nos ensaios de 2001, 2002 e 2003, e o oídio ocorreu apenas em 2000. Para as variáveis ferrugem-da-folha, helmintosporiose, peso de mil sementes (PMS) e rendimento, a análise de variância em todos os experimentos revelou valores de $F$ altamente significativos para os fatores fungicida e cultivar. Houve interação entre os fatores fungicida e cultivar para a ferrugem-da-folha nos anos de 2001, 2002 e 2003, para helmintosporiose nos anos de 2001 e 2003, para o PMS em todos os anos e para o rendimento apenas em 2002 e 2003 (Tabela 1).

No ano de 2000 a helmintosporiose ocorreu em índices de

Tabela 1: Probabilidade de $p$ para o teste $F$ dos fatores e interações para os experimentos conduzidos em 2000, 2001, 2002 , e 2003.

\begin{tabular}{|c|c|c|c|c|c|c|}
\hline Fatores & Anos & Ferrugem-da-folha & Helmintosporiose & Oídio & PMS & Rendimento \\
\hline \multirow[t]{4}{*}{ Fungicida } & 2000 & 0,00001 & - & 0,00001 & 0,00001 & 0,00001 \\
\hline & 2001 & 0,00001 & 0,00001 & - & 0,00001 & 0,00001 \\
\hline & 2002 & 0,00001 & 0,00001 & - & 0,00001 & 0,00001 \\
\hline & 2003 & 0,00001 & 0,00001 & - & 0,00001 & 0,00001 \\
\hline \multirow[t]{4}{*}{ Cultivar } & 2000 & 0,00730 & - & 0,00040 & 0,00001 & 0,02923 \\
\hline & 2001 & 0,01568 & 0,00013 & - & 0,00001 & 0,01095 \\
\hline & 2002 & 0,00001 & 0,00001 & - & 0,00001 & 0,00079 \\
\hline & 2003 & 0,00001 & 0,00003 & - & 0,00001 & 0,00404 \\
\hline \multirow[t]{4}{*}{ Fungicida $\mathrm{x}$ cultivar } & 2000 & 0,35671 & - & 0,02413 & 0,00001 & 0,06514 \\
\hline & 2001 & 0,01568 & 0,02812 & - & 0,03646 & 0,08925 \\
\hline & 2002 & 0,00001 & 0,02752 & - & 0,00001 & 0,00108 \\
\hline & 2003 & 0,00001 & 0,00441 & - & 0,04118 & 0,00230 \\
\hline
\end{tabular}


Tabela 2. Resposta de cultivares de trigo ao controle químico das doenças foliares em Capão Bonito, SP - 2000

\begin{tabular}{|c|c|c|c|c|c|c|c|}
\hline \multirow[t]{2}{*}{$\mathbf{N}^{0}$ tratamento } & \multicolumn{2}{|c|}{$\begin{array}{c}\text { Oídio } \\
\text { Severidade }^{1,5}\end{array}$} & \multirow[t]{2}{*}{$\begin{array}{l}\text { Ferrugem-da-folha } \\
\text { Severidade }^{1,5}\end{array}$} & \multicolumn{2}{|c|}{$\begin{array}{l}\text { PMS } \\
(\mathbf{g})^{2}\end{array}$} & \multirow[t]{2}{*}{$\begin{array}{l}\text { Rendimento } \\
\text { (kg/ha) }\end{array}$} & \multirow[t]{2}{*}{ Aumento relativo $^{3}$} \\
\hline & Com fung. & Sem fung & & Com fung & Sem fung & & \\
\hline 1- IAC 24 & $1,0 \mathrm{a}^{4} \mathrm{~B}(1,0)$ & 2,5 c A $(7,3)$ & $3,5 \mathrm{~b} \quad(14,9)$ & 36,3 bc A & $36,1 \mathrm{ab} A$ & $1557 \mathrm{ab}$ & 19,3 \\
\hline 2 - IAC 289 & 1,0 a B $(1,0)$ & 2,2 c A $(6,1)$ & $3,6 \mathrm{ab} \quad(15,8)$ & $36,7 \mathrm{bc} \mathrm{A}$ & $30,5 \mathrm{~cd} \mathrm{~B}$ & $1538 \mathrm{ab}$ & 55,8 \\
\hline 3 - IAC 350 & 1,5 a B $(2,6)$ & 3,0 bc A $(10,7)$ & $4,9 \mathrm{a} \quad(26,8)$ & 39,6 ab A & $28,3 \mathrm{~d} \mathrm{~B}$ & $1228 \mathrm{~b}$ & 96,2 \\
\hline 4 - IAC 362 & 1,0 a B $(1,0)$ & $4,7 \mathrm{ab} \mathrm{A}(26,3)$ & $4,5 \mathrm{ab} \quad(23,7)$ & 38,4 bc A & $34,1 \mathrm{~b} \mathrm{~B}$ & $1457 \mathrm{ab}$ & 34,4 \\
\hline 5 - IAC 364 & 1,0 a B $(1,0)$ & 3,0 bc A $(11,4)$ & 3,3 b $(13,9)$ & 42,9 a A & 38,4 a B & $1606 \mathrm{a}$ & 10,7 \\
\hline 6 - IAC 370 & 2,0 a B $(4,9)$ & 5,2 a A $(32,2)$ & $4,3 \mathrm{ab} \quad(21,6)$ & 43,0 a A & 33,8 bc B & $1551 \mathrm{ab}$ & 21,9 \\
\hline Com fungicida & $1,2 \mathrm{~B}(1,7)$ & - & $2,4 \mathrm{~B} \quad(6,2)$ & $39,5 \mathrm{~A}$ & - & $1709 \mathrm{~A}$ & 34,6 \\
\hline Sem fungicida & & $3,5 \mathrm{~A}(14,5)$ & $5,8 \mathrm{~A} \quad(37,2)$ & - & $33,6 \mathrm{~B}$ & $1269 \mathrm{~B}$ & - \\
\hline $\mathrm{CV}(\%)$ & & 36,1 & 20,9 & & & 15,3 & \\
\hline
\end{tabular}

${ }^{1}$ Severidade estimada por uma escala de notas de 0-9

${ }^{2}$ Peso de mil sementes

${ }^{3}$ Aumento de rendimento devido à aplicação de fungicida

${ }^{4}$ Médias comparadas pelo teste de Tukey a $5 \%$ de probabilidade. Letras minúsculas comparam as colunas e maiúsculas o fator fungicida.

${ }^{5}$ Porcentagem da área foliar afetada pelas doenças, entre parênteses.

severidade muito baixos em todas as cultivares e por isso a doença não foi considerada nas análises estatísticas. Apesar de baixa precipitação pluviométrica desse inverno, os índices de severidade da ferrugem-da-folha e do oídio foram altos nas parcelas não tratadas. As cultivares IAC 362 e IAC 370 foram as mais afetadas pelo oídio e a cultivar IAC 350 apresentou o maior índice de severidade da ferrugem-da-folha (Tabela 2). O tratamento com o fungicida propiconazole controlou ambas as doenças, independente da cultivar avaliada (Tabela 2). O peso de mil sementes (PMS) de todas as cultivares, com exceção da IAC 24, foi maior nas parcelas tratadas com o fungicida. Nas parcelas sem tratamento, a cultivar IAC 350 apresentou o menor PMS. Sendo essa a cultivar mais afetada pela ferrugem-da-folha, e entre as mais atacadas pelo oídio, evidenciou-se o efeito das doenças sobre essa variável (Tabela 2). Nessa safra o tratamento químico teve menor impacto sobre o rendimento, provavelmente porque as condições climáticas foram pouco favoráveis para a cultura e para o desenvolvimento das doenças, que ocorreram no final do ciclo, período em que houve maior concentração das chuvas.

No ano de 2001 as chuvas ocorreram acima da média normal e foram bem distribuídas durante todo o ciclo, favorecendo a cultura, mas também o desenvolvimento das doenças foliares, que se manifestaram em elevadas severidades (Tabela 3). O tratamento com o fungicida tebuconazole controlou as doenças, sendo muito eficiente para a ferrugem-da-folha, que praticamente não ocorreu nas parcelas tratadas, nas quais não houve diferença entre as cultivares. Apresentaram os menores índices de severidade da helmintosporiose, nas áreas tratadas, as cultivares IAC 350 e IAC 370. A primeira também foi a menos afetada pela doença nas parcelas sem tratamento (Tabela 3). Esse fato pode ter ocorrido porque, como essas cultivares tiveram ciclo mais longo, o tratamento pode ter sido mais preventivo para as mesmas. As cultivares IAC 350 e IAC 364 tiveram os maiores índices de PMS, tanto nas parcelas tratadas quanto nas sem tratamento. Com exceção da cultivar IAC 350, as demais apresentaram maior PMS nas parcelas tratadas. A cultivar IAC 289 teve o maior rendimento e, juntamente com as cultivares IAC 362 e IAC 370, a maior resposta em rendimento ao tratamento químico.

No ano de 2002, por falta de chuvas, a semeadura foi retardada para meados de junho, quando essas foram bem distribuídas e adequadas para a cultura. $\mathrm{O}$ atraso no plantio e a ocorrência de chuvas em seguida, podem ter favorecido o aparecimento antecipado das doenças, especialmente da ferrugem-da-folha, detectada em elevadas severidades nas parcelas sem tratamento. O tratamento químico com tebuconazole, assim como no ano anterior, proporcionou o controle da ferrugem-da-folha e da helmintosporiose (Tabela 4). Nas parcelas tratadas não houve diferença entre os índices de severidade das cultivares para a primeira doença, mas a cultivar IAC 289 foi menos afetada pela helmintosporiose. Nas áreas sem tratamento, as cultivares IAC 289 e IAC 350 foram as menos afetadas por ambas doenças (Tabela 4). Com exceção da cultivar IAC 24, as demais tiveram maior PMS nas parcelas submetidas ao tratamento químico. As cultivares IAC 289 e IAC 350 apresentaram menores valores de PMS na área sem tratamento (Tabela 4). Todas as cultivares responderam com aumento no rendimento quando submetidas ao tratamento químico, sem diferirem entre si, mas as variedades IAC 350 e IAC 370 tiveram aumentos de produção muito expressivos, maiores que $100 \%$ (Tabela 4). 
Tabela 3. Resposta de cultivares de trigo ao controle químico das doenças foliares em Capão Bonito, SP - 2001

\begin{tabular}{|c|c|c|c|c|c|c|c|c|}
\hline \multirow[t]{2}{*}{$\begin{array}{l}\mathrm{N}^{0} \\
\text { tratamento }\end{array}$} & \multicolumn{2}{|c|}{$\begin{array}{c}\text { Ferrugem-da-folha } \\
\text { Severidade }^{1,5}\end{array}$} & \multicolumn{2}{|c|}{$\begin{array}{c}\text { Helmintosporiose } \\
\text { Severidade }^{1,5}\end{array}$} & \multicolumn{2}{|c|}{$\begin{array}{l}\text { PMS } \\
(\mathbf{g})^{2}\end{array}$} & \multirow{2}{*}{$\begin{array}{l}\text { Rendimento } \\
\text { (kg/ha) } \\
\text { Média geral }\end{array}$} & \multirow{2}{*}{$\begin{array}{l}\text { Aumento } \\
\text { relativo }^{3} \\
(\%)\end{array}$} \\
\hline & Com fung. & Sem fung. & Com fung. & Sem fung. & Com fung. & Sem fung. & & \\
\hline 1- IAC 24 & $0,0 \mathrm{a} \mathrm{B}^{4}(0,0)$ & 5,0 a A $(29,8)$ & $3,0 \mathrm{ab} \mathrm{A}(10,0)$ & 8,0 a A $(60,0)$ & $43,9 \mathrm{~b} \mathrm{~A}$ & $37,5 \mathrm{~cd} \mathrm{~B}$ & $2496 \mathrm{~b}$ & 38,7 \\
\hline 2 - IAC 289 & 0,0 a B $(0,0)$ & 3,3 b A $(13,0)$ & 3,0 ab B $(10,0)$ & 7,5 a A $(55,0)$ & $43,3 \mathrm{~b} \mathrm{~A}$ & 35,9 cd B & $3126 \mathrm{a}$ & 69,9 \\
\hline 3 - IAC 350 & 0,0 a B $(0,0)$ & 3,5 b A $(14,6)$ & 2,2 bc B $(6,1)$ & $6,5 \mathrm{~b} A(45,0)$ & $45,9 \mathrm{ab} A$ & 44,0 a A & $2803 \mathrm{ab}$ & 44,7 \\
\hline 4 - IAC 362 & 0,0 a B $(0,0)$ & 5,5 a A $(34,9)$ & 3,2 a B $(12,2)$ & 7,8 a $\mathrm{A}(57,5)$ & $43,0 \mathrm{~b} \mathrm{~A}$ & $35,0 \mathrm{~d} \mathrm{~B}$ & $2683 \mathrm{ab}$ & 88,4 \\
\hline 5 - IAC 364 & 0,0 a B $(0,0)$ & $4,3 \mathrm{ab} \mathrm{A}(21,8)$ & 3,2 a $\mathrm{B}(12,2)$ & 7,8 a $\mathrm{A}(57,5)$ & 48,1 a A & $41,9 \mathrm{ab} B$ & $2549 \mathrm{~b}$ & 44,2 \\
\hline 6 - IAC 370 & 0,0 a B $(0,0)$ & $4,5 \mathrm{ab} \mathrm{A}(24,0)$ & 2,0 c B $(5,0)$ & 7,8 a $\mathrm{A}(57,5)$ & $45,2 \mathrm{ab} \mathrm{A}$ & $39,6 \mathrm{bc} \mathrm{B}$ & $2943 a b$ & 89,7 \\
\hline Com fungicida & $0,0 \mathrm{~B}(0,0)$ & - & $2,8 \mathrm{~B}(9,0)$ & - & $44,9 \mathrm{~A}$ & - & $3456 \mathrm{~A}$ & 62,2 \\
\hline Sem fungicida & & $4,3 \mathrm{~A}(22,6)$ & & $7,5 \mathrm{~A}(55,4)$ & - & $38,9 \mathrm{~B}$ & $2078 \mathrm{~B}$ & - \\
\hline $\mathrm{CV}(\%)$ & & 2,1 & o, & & & & 13,0 & - \\
\hline
\end{tabular}

${ }^{1}$ Severidade estimada por uma escala de notas de $0-9$

${ }^{2}$ Peso de mil sementes

${ }^{3}$ Aumento de rendimento devido à aplicação de fungicida

${ }^{4}$ Médias comparadas pelo teste de Tukey a $5 \%$ de probabilidade. Letras minúsculas comparam as colunas e maiúsculas o fator fungicida.

${ }^{5}$ Porcentagem da área foliar afetada pelas doenças, entre parênteses.

Tabela 4. Resposta de cultivares de trigo ao controle químico das doenças foliares em Capão Bonito, SP - 2002

\begin{tabular}{|c|c|c|c|c|c|c|c|c|}
\hline \multirow[t]{2}{*}{$\begin{array}{l}\mathrm{N}^{0} \\
\text { tratamento }\end{array}$} & \multicolumn{2}{|c|}{$\begin{array}{c}\text { Ferrugem-da-folha } \\
\text { Severidade }^{1,5}\end{array}$} & \multirow[t]{2}{*}{$\begin{array}{l}\text { Helmintosporiose } \\
\text { Severidade }{ }^{1,5}\end{array}$} & \multicolumn{2}{|c|}{$\operatorname{PMS}(\mathrm{g})^{2}$} & \multicolumn{2}{|c|}{$\begin{array}{c}\text { Rendimento } \\
\text { (kg/ha) }\end{array}$} & \multirow{2}{*}{$\begin{array}{l}\text { Aumento }^{3} \\
\text { relativo } \\
(\%)\end{array}$} \\
\hline & Com fung. & Sem fung & & Com fung. & Sem fung & Com fung. & Sem fung & \\
\hline 1- IAC 24 & $2,5 \mathrm{a} \mathrm{B}^{4}(7,3)$ & 7,5 a $\mathrm{A}(55,0)$ & $3,3 \mathrm{ab}(12,6)$ & $37,3 \mathrm{~b} \mathrm{~A}$ & $35,9 \mathrm{ab} \mathrm{A}$ & $2234 \mathrm{~b} \mathrm{~A}$ & 1314 a B & 70,1 \\
\hline 2 - IAC 289 & 2,5 a B $(7,3)$ & $4,8 \mathrm{~b} \mathrm{~A}(27,1)$ & $2,5 \mathrm{c} \quad(7,3)$ & 36,6 b A & 29,9 с B & 2668 a A & 1464 a B & 82,2 \\
\hline 3 - IAC 350 & 2.0 a B $(5,0)$ & $4,3 \mathrm{~b} \mathrm{~A}(22,4)$ & $2,6 \mathrm{c} \quad(7,9)$ & $39,0 \mathrm{~b} \mathrm{~A}$ & 28,2 c B & 2639 a A & 1102 a B & 139,4 \\
\hline 4 - IAC 362 & 2,5 a B $(7,3)$ & 7,3 a A $(52,5)$ & $3,4 \mathrm{ab}(13,8)$ & 37,9 b A & 33,3 b B & $2346 \mathrm{ab} A$ & 1178 a B & 99,3 \\
\hline 5 - IAC 364 & 2,2 a B $(6,1)$ & 7,8 a $\mathrm{A}(57,5)$ & $3,0 \mathrm{bc}(10,3)$ & 42,2 a A & $38,1 \mathrm{a} \mathrm{B}$ & 2103 b A & 1128 a B & 86,4 \\
\hline 6 - IAC 370 & 2,7 a B $(8,6)$ & 8.0 a A $(60,0)$ & 3,8 a $\quad(16,8)$ & $42,3 \mathrm{a} \mathrm{A}$ & 33,6 b B & $2495 \mathrm{ab} A$ & 836 a B & 198,4 \\
\hline Com fungicida & 2,4 B $\quad(6,9)$ & - & $2,6 \mathrm{~B} \quad(7,7)$ & $39,2 \mathrm{~A}$ & - & 2414 A & - & 112,6 \\
\hline Sem fungicida & & 6,7 A $(45,4)$ & $3,6 \mathrm{~A} \quad(15,3)$ & - & $33,2 \mathrm{~B}$ & - & $1170 \mathrm{~B}$ & - \\
\hline $\mathrm{CV}(\%)$ & & & 13,0 & & , I & & & \\
\hline
\end{tabular}

${ }^{1}$ Severidade estimada por uma escala de notas de 0-9

${ }^{2}$ Peso de mil sementes

${ }^{3}$ Aumento de rendimento devido à aplicação de fungicida

${ }^{4}$ Médias comparadas pelo teste de Tukey a 5\% de probabilidade. Letras minúsculas comparam as colunas e maiúsculas o fator fungicida.

${ }^{5}$ Porcentagem da área foliar afetada pelas doenças, entre parênteses. 
Tabela 5. Resposta de cultivares de trigo ao controle químico das doenças foliares em Capão Bonito, SP - 2003

\begin{tabular}{|c|c|c|c|c|c|c|c|c|c|}
\hline \multirow[t]{2}{*}{$\begin{array}{l}\mathrm{N}^{0} \\
\text { tratamento }\end{array}$} & \multicolumn{2}{|c|}{$\begin{array}{c}\text { Ferrugem-da-folha } \\
\text { Severidade }^{1,5}\end{array}$} & \multicolumn{2}{|c|}{$\begin{array}{c}\text { Helmintosporiose } \\
\text { Severidade }^{1,5}\end{array}$} & \multicolumn{2}{|c|}{$\begin{array}{l}\text { PMS } \\
(\mathbf{g})^{2}\end{array}$} & \multicolumn{2}{|c|}{$\begin{array}{c}\text { Rendimento } \\
\text { (kg/ha) }\end{array}$} & \multirow{2}{*}{$\begin{array}{l}\text { Aumento }^{3} \\
\text { relativo } \\
(\%)\end{array}$} \\
\hline & Com fung. & Sem fung & Com fung. & Sem fung & Com fung. & Sem fung & Com fung. & Sem fung & \\
\hline 1- IAC 24 & $3,0 \mathrm{aB}^{4}(10,0)$ & 5,8 a $\mathrm{A}(37,4)$ & 2,0 a B $(5,0)$ & 3,3 abcA(12,2) & $42,2 \mathrm{~b} \mathrm{~A}$ & $33,8 \mathrm{bcd}$ B & 2293 b A & 1078 a B & 112,7 \\
\hline 2 - IAC 289 & 2,0 b B $(5,0)$ & $4,0 \mathrm{~b} \mathrm{~A}(20,0)$ & 2,0 a B $(5,0)$ & $2,8 \mathrm{bcA}(8,6)$ & $41,4 \mathrm{~b} \mathrm{~A}$ & $32,4 \mathrm{~cd} \mathrm{~B}$ & $2135 \mathrm{~b} \mathrm{~A}$ & 1171 a B & 82,3 \\
\hline 3 - IAC 350 & 1,2 c B $(1,7)$ & 3,5 b A $(14,6)$ & 1,0 b B $(1,0)$ & $2,5 \mathrm{cA}(7,3)$ & $45,0 \mathrm{ab} A$ & 39,3 a B & 2606 a b A & 1511 a B & 72,4 \\
\hline 4 - IAC 362 & 2,7 a $B(8,6)$ & 6,0 a A $(40,0)$ & 2,7 a B $(8,6)$ & $3,8 \mathrm{aA}(17,2)$ & $42,0 \mathrm{~b} \mathrm{~A}$ & $31,7 \mathrm{~d} B$ & $1819 \mathrm{~b} \mathrm{~A}$ & 995 a B & 82,8 \\
\hline 5 - IAC 364 & 2,0 b B $(5,0)$ & 6,0 a A $(40,0)$ & 2,0 a B $(5,0)$ & $3,3 \mathrm{abcA}(12,2)$ & 47,3 a A & $37,5 \mathrm{ab} B$ & $2018 \mathrm{~b} \mathrm{~A}$ & 897 a B & 124,9 \\
\hline 6 - IAC 370 & 1,0 c B $(1,0)$ & $6,0$ a $\mathrm{A} 40,0)$ & 1,0 b B $(1,0)$ & $3,5 \mathrm{abA}(14,6)$ & 47,1 a A & 35,6 bc B & 3411 a A & 847 a B & 302,7 \\
\hline Médias & $2,0 \mathrm{~B}(4,6)$ & $5,2 \mathrm{~A}(31,4)$ & $1,8 \mathrm{~B}(3,8)$ & $3,2 \mathrm{~A}(11,8)$ & $44,1 \mathrm{~A}$ & $35,1 \mathrm{~B}$ & $2380 \mathrm{~A}$ & $1083 \mathrm{~B}$ & 119,7 \\
\hline $\mathrm{CV}(\%)$ & 9 & & 16 &, 4 & &, 4 & & & \\
\hline
\end{tabular}

${ }^{1}$ Severidade estimada por uma escala de notas de $0-9$

${ }^{2}$ Peso de mil sementes

${ }^{3}$ Aumento de rendimento devido à aplicação de fungicida

${ }^{4}$ Médias comparadas pelo teste de Tukey a 5\% de probabilidade. Letras minúsculas comparam as colunas e maiúsculas o fator fungicida.

${ }^{5}$ Porcentagem da área foliar afetada pelas doenças, entre parênteses.

Tabela 6. Coeficientes de correlação entre a o peso de mil sementes (PMS) o rendimento e a severidade da ferrugem-da-folha e da helmintosporiose

\begin{tabular}{|c|c|c|c|c|}
\hline Variáveis & Anos & Ferrugem-da-folha ${ }^{1}$ & Helmintosporiose ${ }^{1}$ & Peso de mil sementes ${ }^{2}$ \\
\hline \multirow[t]{4}{*}{ PMS } & 2000 & $-0,67 * *$ & - & - \\
\hline & 2001 & $-0,74 * *$ & $-0,74 * *$ & - \\
\hline & 2002 & $-0,41 * *$ & $-0,31 *$ & - \\
\hline & 2003 & $-0,82 * *$ & $-0,75^{* *}$ & - \\
\hline \multirow[t]{4}{*}{ Rendimento } & 2000 & $-0,66 * *$ & - & $0,68 * *$ \\
\hline & 2001 & $-0,84 * *$ & $-0,84 * *$ & $0,59 * *$ \\
\hline & 2002 & $-0,84 * *$ & $-0,74 * *$ & $0,60 * *$ \\
\hline & 2003 & $-0,82 * *$ & $-0,80 * *$ & $0,76^{* *}$ \\
\hline
\end{tabular}

${ }^{1}$ Coeficientes de correlação para índices de severidade de ferrugem da folha e helmintosporiose obtidos em cada experimento

${ }^{2}$ Níveis de significância pelo Teste t: $(*)$ e $(* *)$ significativo a $5 \%$ e $1 \%$ de probabilidade, respectivamente; n.s. = não significativo

Durante o experimento conduzido em 2003 as chuvas, embora tenham sido escassas, possibilitaram o desenvolvimento da cultura e da ferrugem-da-folha. A helmintosporiose, possivelmente por essa razão, ocorreu em menor intensidade (Tabela 5). Os fungicidas trifloxystrobin+propiconazole controlaram as doenças, sendo mais eficiente nas cultivares IAC 350 e IAC 370. Nas parcelas sem tratamento, as cultivares IAC 289 e IAC 350, assim como no ano anterior, foram as menos atacadas pelas doenças, demonstrando possuir uma relativa resistência. Todas as cultivares apresentaram maior PMS nas parcelas tratadas, mas as cultivares IAC 364, IAC 370 e IAC 350 se destacaram das demais tanto nas áreas tratadas como nas não tratadas. Neste ano as cultivares também apresentaram elevados aumentos relativos no rendimento em resposta ao tratamento, mais de $300 \%$ na cultivar IAC 370 (Tabela 5).

Houve correlação negativa significativa entre a severidade da ferrugem-da-folha e da helmintosporiose e o rendimento da cultura em todos os anos (Tabela 6). Também houve correlação entre essas variáveis e o PMS, mas os valores de $\mathrm{r}^{2}$ foram menores, especialmente no ano de 2002 (Tabela 6). Pode ser que essa última variável tenha sido mais influenciada pelas condições ambientais que prevaleceram na época de enchimento dos grãos e por características inerentes às mesmas.

A ferrugem-da-folha ocorreu em intensidades relativamente 
elevadas nas parcelas sem tratamento em todos os anos, e parece não ter sido muito influenciada pelas condições climáticas. A helmintosporiose, entretanto, foi mais severa em 2001, ano com a maior precipitação. Essa doença mostrou-se mais dependente das condições climáticas, especialmente da precipitação, e esses dados concordam com o constatado por outros autores, ao verificarem que combinações de umidade e temperatura elevadas são responsáveis por severas epidemias dessa doença $(18,21,24)$.

As cultivares não diferiram quanto à severidade das doenças nas parcelas tratadas, com exceção do último ensaio, em 2003, no qual as cultivares IAC 350 e IAC 370 tiveram menores índices de severidade da ferrugem-da-folha e da helmintosporiose e, em 2001 quando tiveram menores índices da helmintosporiose. Como já comentado anteriormente, essas cultivares tiveram ciclo mais longo, podendo por essa razão o tratamento químico ter sido mais preventivo para as mesmas. Nas áreas sem tratamento as cultivares IAC 289 e IAC 350 foram as menos afetadas pelas doenças, demonstrando uma relativa resistência.

Em todos os ensaios houve aumento significativo no PMS em resposta ao tratamento químico. Outros autores já haviam observado que um dos componentes da produção mais influenciados pelo tratamento com fungicidas é o peso e o tamanho dos grãos $(8,10,15)$. Akanda \& Mundt (1) constataram que o peso de sementes foi a variável mais consistente e negativamente influenciada pela ferrugem da folha. Os aumentos no tamanho e peso dos grãos parecem ser devidos não só ao controle de doenças, mas também à maior translocação de nutrientes e ao aumento no período de maturação proporcionados pelos fungicidas $(10,15)$. No presente trabalho houve correlação significativa entre o PMS e a severidade das doenças (Tabela 6), mas diferente comportamento das cultivares com relação a essa característica. Nas áreas sem tratamento a cultivar IAC 364 apresentou alto PMS em todos os anos, seguido das cultivares IAC 24 e IAC 350. Como a primeira cultivar apresentou elevados índices de severidade das doenças em praticamente todos os experimentos, o PMS elevado pode ser uma característica inerente à mesma. Em trabalhos anteriores, a maior resposta em aumento no tamanho e peso dos grãos ao tratamento químico foi devida, principalmente, ao controle de doenças, ou seja, cultivares mais suscetíveis apresentaram as maiores respostas ao controle químico quanto a essas variáveis $(8,15)$. Nos presentes experimentos, a cultivar IAC 364 parece ter demonstrado a característica de maior PMS que as demais. As cultivares IAC 370, IAC 350 e IAC 289 apresentaram grandes respostas em PMS, provavelmente porque para as mesmas o tratamento químico foi mais preventivo, já que elas tiveram um ciclo mais longo.

Em todos os anos o tratamento químico proporcionou retorno em rendimento. Em 2000 as condições climáticas foram pouco favoráveis para cultura, que ainda assim respondeu à aplicação de fungicidas. O retorno foi elevado nos três últimos experimentos, mas maior em 2002 e 2003, anos em que as condições climáticas foram mais adequadas para a cultura, confirmando o fato de que condições favoráveis para a obtenção de elevadas produtividades são as que justificam o tratamento com fungicidas $(17,22)$. No presente trabalho, entretanto, o controle químico também foi vantajoso nos anos menos favoráveis para a cultura, como 2000 e 2001, provavelmente porque nessa região as condições climáticas são propícias ao desenvolvimento de doenças foliares.

As cultivares estudadas responderam positivamente em rendimento ao controle químico, mas apresentaram respostas variáveis de acordo com o ano do experimento, embora tenha havido correlação negativa significativa entre a incidência de doenças e o rendimento (Tabela 6). As cultivares IAC 370 e IAC 289 estiveram entre as com maior aumento em rendimento em três experimentos, as cultivares IAC 350, IAC 362 e IAC 364 em dois, e a cultivar IAC $24 \mathrm{em}$ um. Trabalhos anteriores obtiveram as maiores respostas em rendimento ao tratamento químico nas cultivares mais suscetíveis às doenças foliares $(2,6,8,11,14,15)$. No presente trabalho as cultivares IAC 289 e IAC 350 apresentaram elevada resposta em rendimento ao tratamento químico, embora estivessem entre as menos afetadas pelas doenças nas áreas sem tratamento. Além das doenças, provavelmente outros eventos contribuíram para o retorno em rendimento, tais como o ciclo e o potencial produtivo da cultivar. Também pode ser que o nível de resistência que essas cultivares exibiram não seja suficiente para gerar uma grande diferença na resposta em relação às demais cultivares, mais suscetíveis. As cultivares IAC 370, IAC 350 e IAC 289 apresentaram em média, ciclo mais longo (67 a 69 dias até o florescimento) que as demais (59 a 61 dias), e por essa razão podem ter demonstrado as maiores respostas em rendimento, especialmente porque o período de maturação é um importante componente do rendimento $(10,15)$. As plantas de ciclo mais longo, que ficam mais tempo no campo, são mais expostas às doenças, como já havia sido observado por Barros (6). O grande impacto que o tratamento químico teve sobre o rendimento no último ano pode ter ocorrido em parte porque foi utilizado trifloxystrobin + propiconazole, um fungicida formulado com um triazol e uma estrubilurina. Cook et al. (9) verificaram que a aplicação de fungicidas, especialmente do grupo das estrubilurinas, pode prolongar o ciclo da cultura, ou seja, o período em que a cultura mantêm a área foliar verde, e esse fato parece ter impacto positivo sobre o rendimento.

Neste trabalho, verificou-se que as cultivares de trigo recomendadas para o Estado de São Paulo apresentam uma grande resposta em rendimento e PMS ao tratamento químico. Essa resposta pode ser maior em anos com condições climáticas mais adequadas para a cultura e em cultivares de ciclo mais longo.

\section{REFERÊNCIASBIBLIOGRÁFICAS}

1. Akanda, S.I.; Mundt, C.C. Effect of two-components mixture and yellow rust on yield components of wheat. Plant Pathology, Cambridge, v.46, n. 4, p.566-580, 1997.

2. Barcellos, A. L.; Ignaczac, J.C. Efeito da ferrugem da folha em diferentes estágios de desenvolvimento do trigo. In: Reunião Anual Conjunta de Pesquisa de Trigo, 10., 1978, Porto Alegre. Solos e Técnicas Culturais, Economia e Sanidade. Passo Fundo: Embrapa, Cnptrigo, 1978. p.212-219.

3. Barcellos, A. L.; Moraes-Fernandes, M.I.B.; Roelfs, A.P. Ferrugem da folha do trigo (Puccinia recondita): durabilidade da resistência. Summa Phytopathologica, Jaboticabal, v.23, n.2, p.101-117, 1997.

4. Barros, B.C.; Castro, J.L.; Fregonezi, L. E. Efeito de época de aplicação de fungicidas no rendimento do trigo. Biológico, São Paulo, v.50, n. 8, p.181-186, 1984.

5. Barros, B.C. Trigo/Brasil: O papel das doenças na baixa produtividade. Correio Agrícola, São Paulo, v.2, p.703-710, 1985. 
6. Barros, B.C. Avaliação da resistência varietal e da resposta de cultivares de trigo (Triticum aestivum $\mathrm{L}$.) ao controle químico de oídio, mancha marrom e ferrugem da folha. 1988. 122p. Tese (Doutorado em Fitopatologia) - Escola Superior de Agricultura "Luiz de Queiroz", Universidade de São Paulo, Piracicaba, 1988.

7. Barros, B.C.; Salgado, C.L.; Oliveira, D.A ; Ruiz, E.L.A. Efeito da inoculação artificial de Heminthosporium sativum (PAM.) King \& Bakke em plantas de trigo em diferentes estádios de desenvolvimento. Arquivos do Instituto Biológico, São Paulo, v.56, n.1/2, p.75-77, 1989.

8. Bockus, W.W.; Bowden, R.L.; Claassen, M.M.; Gordon, W.B.; Heer; W.F.; Shroyer, J.P. Time of application and winter wheat genotype affect production of large seed after fungicide application. Canadian Journal of Plant Science, Ottawa, v. 77, p.567-572, 1997.

9. Cook, R.J.; Hims. M.J.; Vaughan, T.B. Effects of fungicide spray timing on winter disease control. Plant Pathology, Cambridge, v.48, p.33-50, 1999.

10. Dimmock, J.P.R.E.; Gooding, M.J. The effect of fungicides on rate and duration of grain filling in winter wheat in relation to maintenance of flag leaf green area. Journal of Agricultural Science, Cambridge, v.138, p.1-16,2002.

11. Fernandes, J.M.C.; Vieira, J.C.; Picinini, E.C.; Sartori, J.F.; Prestes. A.M. Resposta de cultivares de trigo ao tratamento com fungicidas. In: Reunião Nacional de Pesquisa de Trigo, 11, 1980, Porto Alegre. Sanidade. Passo Fundo: Embrapa, Cnptrigo, 1980. p.56 -75.

12. Fernandes, J.M.C.; Rosa, O.S.; Picinini, E.C. Perdas no potencial de rendimento de linhas quase isogênicas de trigo devido ao oídio. Fitopatologia Brasileira, Brasília, v.13, n.2, p.131-131, 1988. (Resumo).

13. Fernandes, J.M.C.; Picinini, E.C. Sistema de suporte à tomada de decisão para otimização do uso de fungicidas na cultura do trigo. Fitopatologia Brasileira, Brasília, v.24, n.1, p.9-17, 1999.

14. Giordani, N. A.; Neto, N. Resposta de cultivares e linhagens de trigo a fungicidas - 1985. In: Reunião Nacional de Pesquisa de Trigo, 14, 1986, Londrina. Resumos. Londrina: Iapar, 1986. p.88.

15. Gooding, M.J.; Dimmock, J.P.R.E.; France, J.; Jones, S.A. Green leaf area decline of wheat flag leaves: the influence of fungicides and relationships with mean grain weight and grain yield. Annals Applied Biology, Wellesbourne, v.136, p.77-84, 2000.

16. Goulart, A.C.P. Transmissão de Bipolaris sorokiniana de sementes de trigo ao coleóptilo de trigo. Fitopatologia Brasileira, Brasília, v.22, n.1, p.5-8, 1996.
17. Goulart, A.C.P.; Paiva, F.de A.; Melo Filho, G.A.; Richetti, A. Controle de doenças da parte aérea do trigo pela aplicação de fungicidas - viabilidade técnica e econômica. Summa Phytopathologica, Jaboticabal, v. 24, n.2, p.160-167, 1998.

18. Hetzler, J.; Eyal, Z.; Mehta, Y.R.; Campos, L.A.C; Fehrmann, H.; Kushnir, U.; Oren, J.Z.; Cohen, L. Interaction between Cochliobolus sativus and wheat cultivars. In: Saunders, D.A. (Ed.) Proc. Int. Conf. On Wheat for Non-Traditional Warmer Areas, Cimmyt: Mexico, 1991. p.146-164.

19. Linhares, W.I. Perdas de produtividade ocasionadas por oídio na cultura de trigo. Fitopatologia Brasileira, Brasíllia, v.13, n.1, p.74-75, 1988.

20. Mehta. Y.R.; Riede, C.R.; Campos, L.A C.; Kholi, M.M. Integrated management of major wheat diseases in Brazil: an example for the Southern Cone region of Latin America. Crop Protection, New York, v.11, p.517-524, 1992.

21. Mehta. Y.R. Manejo integrado de enfermedades del trigo. Santa Cruz de la Sierra, Imprenta Landivar, 1993. 314 p.

22. Recomendações da Comissão Técnica de Trigo para 2002. Boletim Técnico Iac, Campinas, n.167, 2002. 92 p.

23. Reis, E.M.: Fernandes, J.M.C.; Piccinini, E.C. Estratégias para o controle de doenças do trigo. Documentos Embrapa-Cnptrigo, Passo Fundo, n.7, 1988. 50p.

24. Reis, E.M. Previsão de doenças de plantas. Universitária: Passo Fundo, 2004. 316 p.

25. Reis, E.M.; Forcelini, C.A. Transmissão de Bipolaris sorokiniana para órgãos radiculares e aéreos do trigo. Fitopatologia Brasileira, Brasília, v. 18, p.76-81, 1993.

26. Reis, E.M.; Silva, C.L.; Casa, R.T.; Medeiros, C.A. Decomposição dos restos culturais do trigo e sobrevivência saprofítica de Bipolaris sorokiniana. Fitopatologia Brasileira, Brasília, v.23, n.1, p.6264, 1998.

27. Reis, E.M.; Casa, R.T.; Hoffman, L.; Mendes, E.M. Effect of leaf rust on wheat grain yield. Fitopatologia Brasileira, Brasília, v.25, n.1, p.67-71, 2000.

28. Trigo - Agrianual 2003: Anuário da Agricultura Brasileira. São Paulo, p.479-487, 2003.

29. Triller, C.; Mehta, Y.R. Efeito da idade da folha bandeira de trigo na expressão de resistência a Bipolaris sorokiniana. Summa Phytopatologica, Jaboticabal, v. 23, n.2, p.167-169, 1997.

30. Zadoks, J.C.; Chang, T.T.; Konzak, C.F. A decimal code for the growth stages of cereals. Weed Research, Oxford, v.14, p.415421, 1974. 